\title{
Kainic Acid-Induced Neuronal Degeneration in Hippocampal Pyramidal Neurons Is Driven by Both Intrinsic and Extrinsic Factors: Analysis of FVB/N $\leftrightarrow$ C57BL/6 Chimeras
}

\author{
Li Liu, ${ }^{1}$ Kristin M. Hamre ${ }^{1}$, and Dan Goldowitz ${ }^{1,2}$ \\ ${ }^{1}$ Department of Anatomy and Neurobiology, University of Tennessee Health Science Center, Memphis, Tennessee 38163, and ${ }^{2}$ Department of Medical \\ Genetics, Centre for Molecular Medicine and Therapeutics, Child and Family Research Institute, University of British Columbia, Vancouver, British \\ Columbia, V5Z 4H4, Canada
}

The excitotoxic effects of kainic acid (KA) in the mouse hippocampus is strain dependent. Following KA administration, the large majority of hippocampal pyramidal cells die in the FVB/N (FVB) mouse, while the pyramidal cells of the C57BL/6 (B6) strain are largely spared. We generated aggregation chimeras between the sensitive FVB and the resistant B6 strains to investigate whether intrinsic or extrinsic features of a neuron confer cell vulnerability or resistance to KA. The constitutive expression of transgenic green fluorescence protein (GFP) or $\beta$-galactosidase expressed from the ROSA26 locus was used to mark cells in FVB or B6 mice, respectively. These makers enable the identification of cells from each parental genotype while TUNEL (terminal deoxynucleotidyl transferase-mediated biotinylated dUTP nick end labeling)-staining labeled dying cells. The analysis of the percentage of dying cells in FVB-GFP $\leftrightarrow$ B6-ROSA chimeras yielded an intriguing mix of both intrinsic and extrinsic factors in the readout of cell phenotype. Thus, normally resistant B6-ROSA pyramidal neurons demonstrated an increasing sensitivity to KA, in a linear fashion, when the percentage of FVB-GFP cells was increased, either across chimeras or in different regions of the same chimera. However, the death of B6-ROSA pyramidal cells never exceeded $\sim 70 \%$ of the total amount of B6 neurons regardless of the amount of FVB cells in the chimeric hippocampus. In a similar manner, FVB-GFP cells show lower amounts of cell death in chimeras that are colonized by B6-ROSA cells, but again, are never fully rescued. These data indicate that both intrinsic and extrinsic factors modulate the sensitivity of hippocampal pyramidal cells to kainic acid.

\section{Introduction}

Neuronal loss is one of the key underlying features of the debilitating effects of several neurological disorders including stroke, epilepsy, Parkinson disease, and Huntington disease, although the causative factors in these various neuronal degenerative states are not well understood. The genetic endowment of a neuron and a neuron's environment are two major factors that determine susceptibility to insults that cause neurodegeneration.

Kainic acid (KA) is a neuroexcitotoxin that induces seizures and hippocampal pyramidal neuron death, and is a commonly used model system for the investigation of hyperactivity-induced neuronal degeneration. Differential sensitivity of hippocampal neurons to kainic acid has been well documented (Ferraro et al.,

\footnotetext{
Received Dec. 27, 2011; revised May 23, 2012; accepted June 23, 2012.

Author contributions: L.L. and D.G. designed research; L.L. performed research; L.L., K.M.H., and D.G. analyzed data; L.L., K.M.H., and D.G. wrote the paper.

This work was supported by a Canadian Institutes of Health Research Operating Grant. The statistical assistance of Drs. Jim Wan and Scott Heldt is gratefully acknowledged.

The authors declare no competing financial interests.

Correspondence should be addressed to either Dan Goldowitz or Li Liu at the above address. E-mail: dang@cmmt.ubc.ca or lliu8@uthsc.edu.

DOI:10.1523/JNEUROSCI.6478-11.2012

Copyright $\odot 2012$ the authors $\quad 0270-6474 / 12 / 3212093-09 \$ 15.00 / 0$
}

1995; Schauwecker and Steward, 1997; McKhann et al., 2003). Furthermore, it has been found that the genetic background of laboratory rodents are important determinants of their response to many kinds of experimental lesions (Schauwecker and Steward, 1997; Schori et al., 2002; Liu et al., 2003). Schauwecker and Steward (1997) demonstrated that certain commonly used strains of mice, specifically $\mathrm{C} 57 \mathrm{BL} / 6$ and $\mathrm{BALB} / \mathrm{c}$, are resistant to KA-induced neuronal death in pyramidal cells of the hippocampus compared with the highly susceptible pyramidal neurons in the FVB/N and 129/SvEms strains. However all mice exhibited similar levels of seizure activity (Schauwecker and Steward, 1997; Schauwecker, 2002).

The cell intrinsic and extrinsic underpinnings of this differential sensitivity to neurotoxic agents are currently under investigation. A cell intrinsic mechanism is indicated by studies that show that KA's neurotoxic effect is receptor-mediated (Ogita et al., 2003, 2005; Lopez-Picon et al., 2006). Evidence for an extrinsic effect comes from the involvement of glia, particularly microglia, in the death of pyramidal neurons following KA (Hong et al., 2010; Zhu et al., 2010).

The technique of experimental mouse chimeras is an ideal in vivo model to explore whether the driving influences on cell phe- 

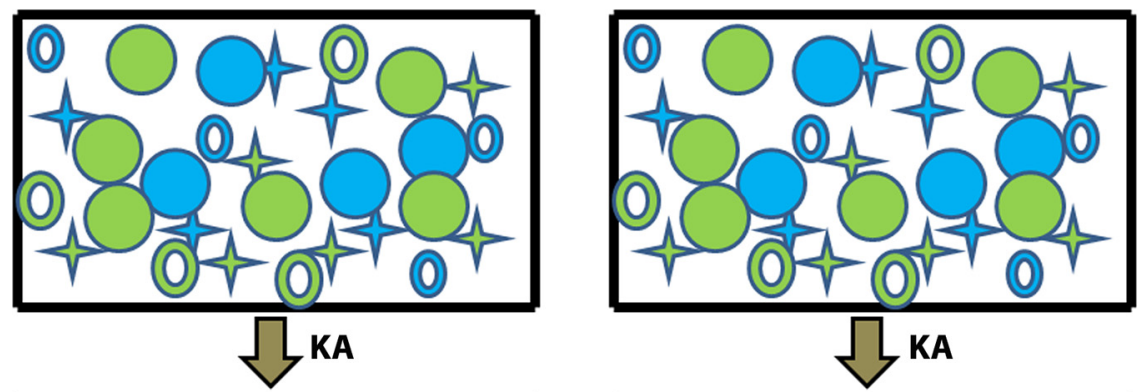

KA
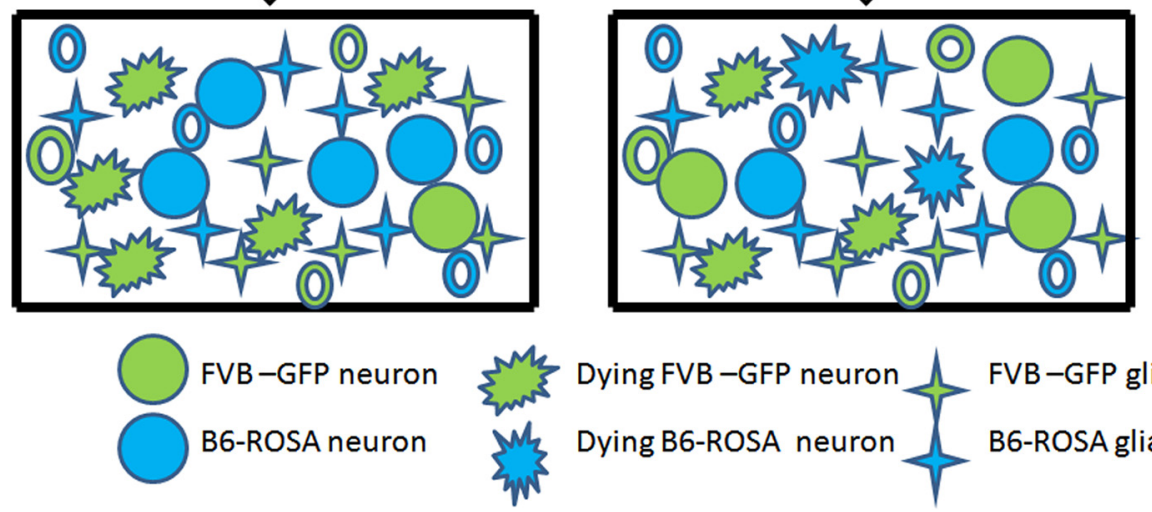

Dying FVB-GFP neuron
Dying B6-ROSA neuron

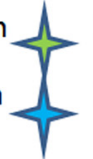

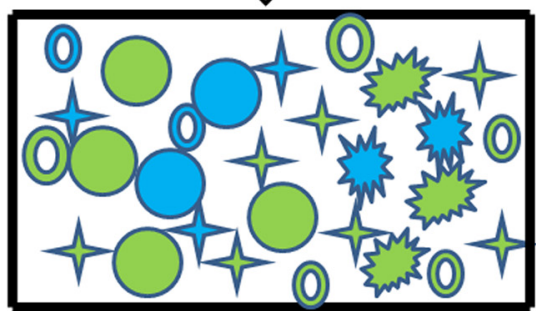

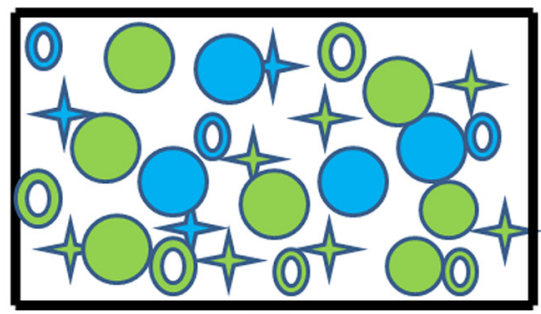

KA

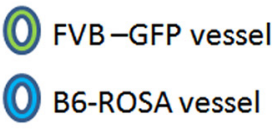

Figure 1. Schema of outcomes and conclusions from a chimera experiment. This diagram shows three possible outcomes (top) in the chimeric hippocampus following kainic acid injection (bottom). (1) A cell intrinsic model would be supported by results wherein the neurons from the sensitive strain (FVB-GFP, green) show cell death while the majority of the neurons from the resistant strain (B6-ROSA, blue) survive. (2) A cell extrinsic model, where some outside factor other than cell genotype is determining survival and death would be supported if neurons from both genotypes have both phenotypes after KA treatment, as illustrated in the middle. (3) A mixed model would be supported if there is a combination of intrinsic and extrinsic factors that interact to determine the effects of kainate on neurons. For example, glia or endothelial cells from the resistant (blue) or sensitive (green) genotypes help to determine whether neurons of both genotypes survive or die. However, in this model, there is also the intrinsic resistance to death in the insensitive cells and innate susceptibility to death in the sensitive cells.

notype are cell-autonomous or non-cell-autonomous. Figure 1 presents, in diagram form, 3 idealized scenarios or predictions by which KA-resistant neurons $(\mathrm{C} 57 \mathrm{BL} / 6 \mathrm{~J})$ and KA-sensitive neurons $(\mathrm{FVB} / \mathrm{N})$ might respond to $\mathrm{KA}$ in the chimeric setting. In a cell-intrinsic mechanism, the phenotypic readout is in line with cell genotype and would yield pyramidal cell death only in sensitive cells, while resistant cells survive; the phenotypic readout is true to the genotypic make-up of each cell (\#1). However, if an environmental factor is responsible for cell death, an example of a cell extrinsic model, then both genotypes would respond similarly within the chimeric environment (\#2). A mixed model of intrinsic and extrinsic effects is illustrated in example \#3 where other factors or cell types in the neuropil, such as glia, provide an extrinsic input to neurons and interact with cell intrinsic features of the neuron to result in increased cell death of some resistant neurons and increased survival of some sensitive neurons. Cleveland and colleagues used the chimeric approach to identify a cell-extrinsic component to neuronal death in a mouse model of amyotrophic lateral sclerosis (Clement et al., 2003). The aim of the present study is to explore the cellular basis of neuroexcitoxicity caused by KA using experimental mouse chimeras.

\section{Materials and Methods}

Production of experimental mouse chimeras

Adult FVB/N-GFP [FVB.Cg-Tg (CAG-EGFP) B5Nagy/J, stock number 003516, abbreviated as FVB-GFP] and C57BL/6J-ROSA26 [B6.129S7-Gt (ROSA) 26Sor/J, stock number 002192, abbreviated as B6-ROSA] mice were purchased from The Jackson Laboratory. FVB-GFP mice express GFP (green fluorescence protein) under a chicken $\beta$-actin promoter. GFP has robust expression in most cell types. B6-ROSA mice express $\beta$-galactosidase ( $\beta$-gal) in almost all tissues of the developing embryo and adult mouse due to the insertion of the lac $Z$ gene into a constitutively expressed locus (Soriano, 1999). These two cells makers enable us to identify the cells from each parental strain. Three mice from each of the lines were used for controls and 27 chimeras were produced as previously described (Mullen and Whitten, 1971; Goldowitz et al., 1992). Briefly, 5to 8-week-old B6-ROSA and FVB-GFP females were injected with Pregnant Mare Serum (4-6 IU, Sigma-Aldrich) followed in 40-44 h with a similar amount of Human Chorionic Gonadotropin (Sigma-Aldrich) to superovulate the females in preparation for mating with males of the same genotype. Four- to eight-cell embryos were collected from females with vaginal plugs (indicating successful copulation) $2 \mathrm{~d}$ after mating. One embryo from each genotype, after removal of the zona pellucida with gentle Pronase treatment, was then aggregated with an embryo of the other genotype in individual wells created by indentations formed using a Hungarian darning needle (Hadjantonakis et al., 1998). The aggregated pairs of embryos were then cultured overnight and embryos that successfully fused were implanted into the uterine horns $(\sim 10-12$ chimeric embryos in each uterine horn) of $2.5 \mathrm{~d}$ pseudo-pregnant ICR female hosts. Donor females were carefully monitored and the number of births was recorded for each female.

\section{Kainic acid treatment}

Kainic acid (30 mg/kg total; Ocean Produce International) was dissolved in isotonic, sterile saline, $\mathrm{pH} 7.4$, and administered subcutaneously in two injections of 20 and $10 \mathrm{mg} / \mathrm{kg}$ separated by $30 \mathrm{~min}$. This dosage was calibrated in the FVB and B6 parental strains and examined at different survival times to optimize histological detection of cell death and cell genotype. Previous studies have shown differential levels of cell death at different survival times following the KA insult (Hu et al., 1998; Schauwecker, 2002; McKhann et al., 2003). However, results show that the level of TUNEL (terminal deoxynucleotidyl transferase-mediated biotinylated dUTP nick end labeling)-labeled cell death at $48 \mathrm{~h}$ is higher than at $24 \mathrm{~h}$ and $7 \mathrm{~d}$ (Hu et al., 1998). At $24 \mathrm{~h}$, the level of TUNEL ${ }^{+}$cells was limited while at $7 \mathrm{~d}$, the genotypic marker was lost (data not shown); thus chimeras were processed at the $48 \mathrm{~h}$ time point. A total of 27 adult (2- to 3-month-old) chimeras, both males and females, were used for these studies. Twenty-four chimeras received subcutaneous injections of KA 

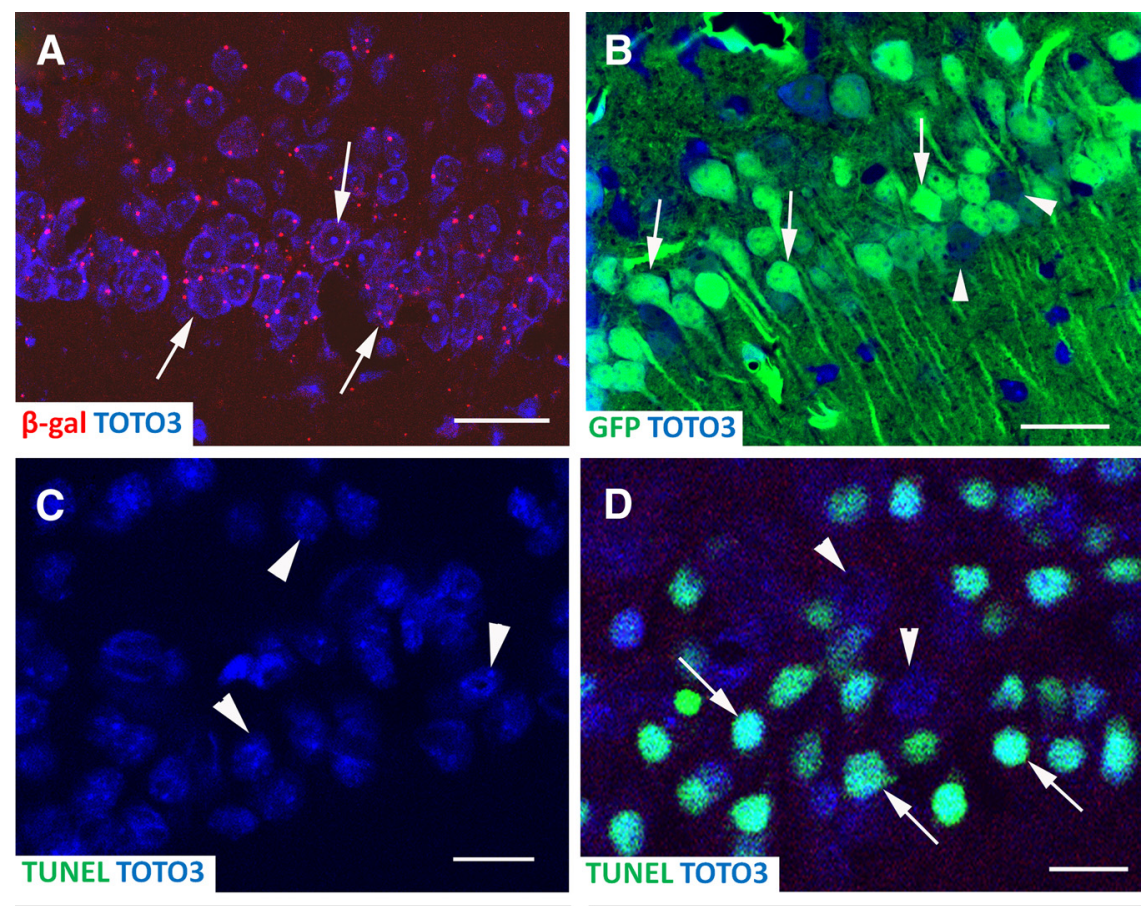

\section{E TUNEL ${ }^{+}$cells after KA lesion}

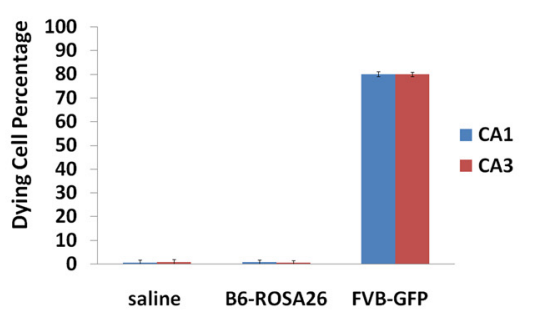

Figure 2. Histological analyses identify the cell genotype and cell death phenotype in response to KA in control hippocampi. $\boldsymbol{A}$, Immunostaining with anti- $\beta$-galactosidase antibody demonstrates the labeling (arrows, red puncta surrounding nuclei stained blue by TOT03) of virtually all neurons in the CA1 region of the hippocampus of a B6-ROSA mouse. Pyramidal cells from the B6-ROSA mouse have $2-4$ red puncta around the nucleus ( $\boldsymbol{A}$, arrows). $\boldsymbol{B}$, Section from an FVB-GFP transgenic mouse shows GFP expression (green, arrows) with nuclei stained with TOT03 (blue). Almost all pyramidal neurons in the CA1 region are GFP positive (examples marked by arrows) in the hippocampus of FVB-GFP mice. Only a few neurons, most with a polymorphic shape, showed no or limited GFP expression (only TOTO3 positive, arrowheads). C, No TUNEL ${ }^{+}$cells are seen in the hippocampus of the B6-ROSA mouse $48 \mathrm{~h}$ after injection with KA. $\boldsymbol{D}$, Numerous TUNEL ${ }^{+}$cells are found in the FVB-GFP mouse CA1 region with the same KA regimen. $\boldsymbol{E}$, A baseline level of cell death in response to KA was determined for the FVB-GFP and B6-ROSA neurons in the CA1 and CA3 regions in control animals. About $80 \%$ of the pyramidal cells are TUNEL ${ }^{+}$in the FVB-GFP hippocampus $48 \mathrm{~h}$ after KA administration while almost no B6-ROSA cells are TUNEL ${ }^{+}$. $\boldsymbol{F}$, To determine the fidelity of marking dying cells with the GFP marker at $48 \mathrm{~h}$ post-kainic acid injection, we calculated the percentage of cells in the FVB-GFP hippocampus that were colabeled with TUNEL. About $95 \%$ of the TUNEL ${ }^{+}$cells were colabeled by the GFP genetic marker. Scale bar, $50 \mu \mathrm{m}$.

and three were used as controls. Mice typically started to seize $\sim 15$ min after the first injection and were monitored continuously for $4 \mathrm{~h}$ for the extent of seizure activity. Seizures were rated according to a previously defined scale (Racine, 1972; Schauwecker and Steward, 1997): stage 1, immobility; stage 2, forelimb and/or tail extension, rigid posture; stage 3, repetitive movements, head bobbing; stage 4 , rearing and falling; stage 5 , continuous rearing and falling; stage 6 , severe tonic-clonic seizures. A minimum of $1 \mathrm{~h}$ of grade 5 seizures was taken as the threshold for inclusion in the analysis. Two chimeras had seizures that did not last $>1 \mathrm{~h}$ and these were not processed further. Another six chimeras died from this procedure, a mortality rate that was the same in our analysis of KA injections in B6 and FVB strains. We followed this standard for determining the chimeras that were used for detailed histological analysis, cognizant of the fact, however, that chimeras represented unique organisms and individual variances may present unusual opportunities to understand mechanisms associated not only with cell death, but seizures and related phenomena. All animal handling and treatments were performed in accordance with $\mathrm{NIH}$ animal care guidelines and were performed under a University of Tennessee Health Science Center Institutional Animal Care and Use Committee-approved animal protocol.

At $2 \mathrm{~d}$ after KA injection, the chimeras that survived and met criteria $(n=19$, including the three controls) were perfused, under deep Avertin anesthesia, with $4 \%$ paraformaldehyde for $15 \mathrm{~min}$ followed by a $30 \mathrm{~min}$ immersion in the same fixative, and then transferred to $0.1 \mathrm{M}$ PBS. Brains were then removed and cryoprotected in 30\% sucrose in PBS before sectioning in the horizontal plane on a cryostat. Serial, $25 \mu \mathrm{m}$ sections were saved as soon as the ventral portion of the hippocampal formation appeared.

\section{Processing of tissue for analysis}

TUNEL staining. Sections were processed using a Millipore Bioscience Research Reagents kit for the identification of nicked or damaged DNA. Sections were rinsed in PBS, treated with $1 \%$ proteinase $\mathrm{K}$ for $8 \mathrm{~min}$ at room temperature, and then incubated with equilibration buffer for $10 \mathrm{~min}$, followed by $\mathrm{TdT}$ overnight $\left(4^{\circ} \mathrm{C}\right)$. Sections were incubated with anti-digoxigenin antibody conjugated with fluorescein or rhodamine for $1 \mathrm{~h}$ at room temperature. Thymus tissue provided in the kit was run as a positive control. The localization of TUNEL to the nucleus allows for accurate counting of dead or dying cells and costaining with cytoplasmic antigens (see below). TUNEL in green or red allowed us to double- or triple-label neurons by immunostaining with cell genotype markers, e.g., anti-GFP or - $\beta$-gal.

Immunohistochemical detection of cell genotype and double labeling for cell death phenotype. $\mathrm{FVB} / \mathrm{N}$ mice are transgenic for a GFP reporter gene and GFP-positive cells can be visualized unstained using fluorescence illumination or using anti-GFP immunocytochemistry. This latter procedure was used to visualize $\mathrm{GFP}^{+}$and $\mathrm{TUNEL}^{+}$double-labeled neurons. C57BL/6 mice with the ROSA26 genetrap construct have cellular expression of $\beta$-galactosidase (X-gal), which can be visualized with a simple histochemical stain for $\beta$-gal (Jensen et al., 2004) or immunocytochemically using an antibody to $\beta$-gal.

For double-labeling, the TUNEL protocol was followed by immunostaining with antibodies against $\beta$-gal and/or GFP to identify cell genotype. Briefly, the sections were incubated in $1 \%$ bovine serum albumin and $0.3 \%$ Triton X-100 (Sigma) in PBS for $1 \mathrm{~h}$ at room temperature, followed by incubation with goat anti- $\beta$-gal (1:50, Biogenesis Inc) and/or mouse anti-GFP primary antibody (1:500, Invitrogen Inc.) overnight at $4^{\circ} \mathrm{C}$. Following rinses in PBS, the sections were incubated in a rabbit antigoat conjugated with Alexa Fluor 594 or Alexa Fluor 647 secondary antibody (1:400, Invitrogen Inc., for $\beta$-gal) and/or horse anti-mouse secondary antibody (Alexa Fluor 488 or Alexa Fluor 555, 1:400, Invitrogen Inc., for GFP) for $1 \mathrm{~h}$ at room temperature. After rinsing in PBS, sections were counterstained with a nuclear marker, TOTO3 (2 $\mu \mathrm{M}$, Invitrogen Inc) to label all cell nuclei for $5 \mathrm{~min}$ at room temperature and coverslips applied using an anti-fade reagent (Invitrogen). Material was then ready for observation and analysis using a Bio-Rad Confocal microscope. 

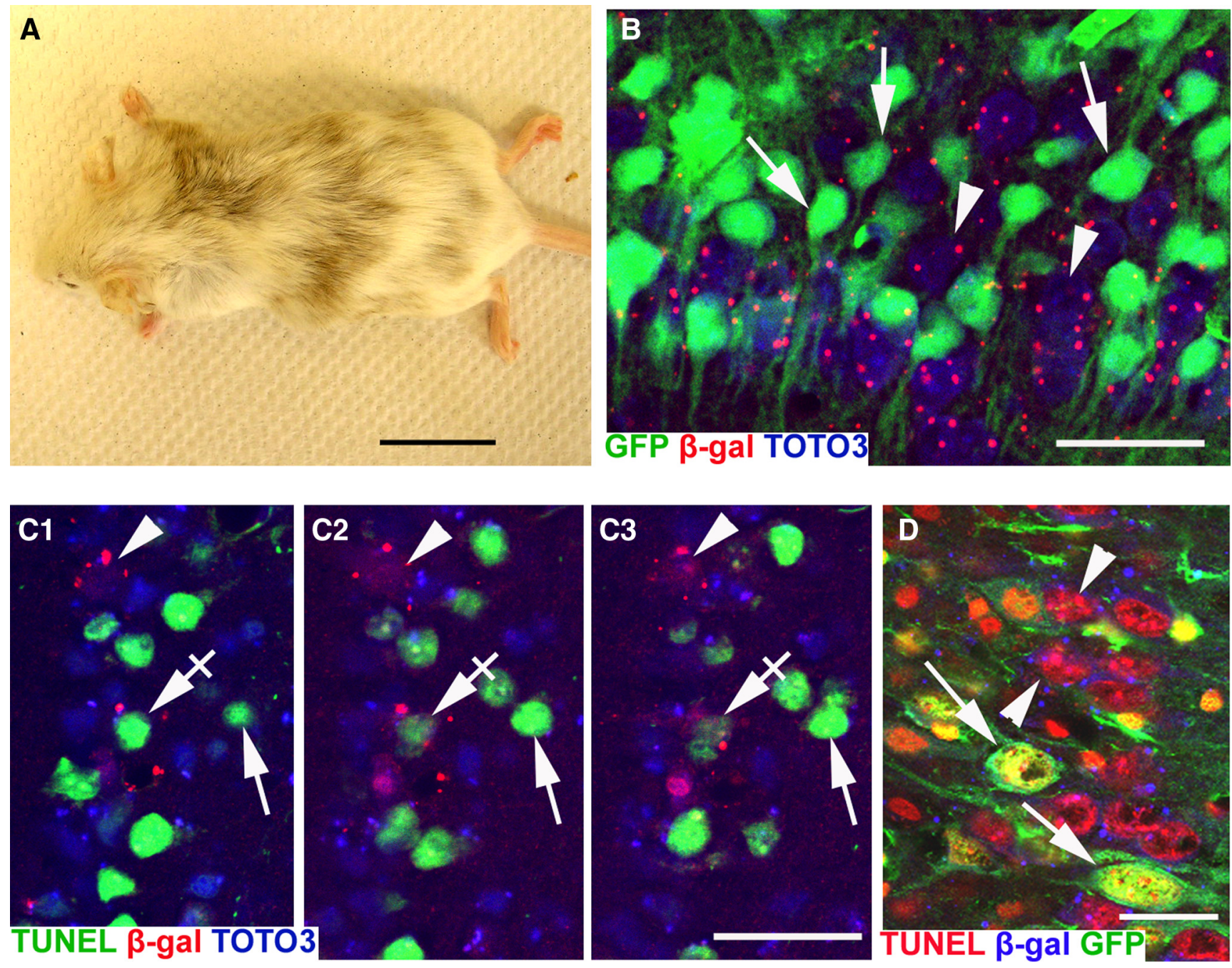

Figure 3. Analysis of kainic acid-induced cell death in the hippocampus of chimeric mice. B6-ROSA pyramidal cells can undergo cell death. A, A B6-ROSA $\leftrightarrow$ FVB-GFP chimeric mouse is shown to illustrate the differing coat colors as an overt sign of chimerism. $\boldsymbol{B}$, Histological image from a control B6-ROSA $\leftrightarrow$ FVB-GFP chimeric mouse. GFP-fluorescence (green) marks FVB pyramidal cells (arrows) while anti- $\beta$-gal immunocytochemistry (red) labels B6-ROSA neurons (arrowheads). TOTO3 (blue) stains all nuclei from both FVB-GFP and B6-ROSA strains. All pyramidal cells are labeled with one of the genotypic markers, indicating that cell marker staining accounts for all neurons in the chimeric cerebellum. Thus, when only one genotype marker is used, TOT03 highlights the cells of the other genotype (C1-C3). C, Double immunostaining for anti- $\beta$-gal (red puncta) and TUNEL (green) in a B6-ROSA $\leftrightarrow$ FVB-GFP chimera ( $\sim 40 \%$ B6-ROSA) $48 \mathrm{~h}$ after kainic acid injection. As expected, TUNEL ${ }^{+}$cells are found in FVB-GFP cells (green only, arrow). However, a TUNEL ${ }^{+}$B6-ROSA cell (green with surrounding red puncta, crossed arrowheads) is also seen. This cell is followed in serial sections (C1-C3) to confirm the presence of associated $\beta$-gal-positive puncta and confirm the genotype as B6-ROSA. A TUNEL-negative B6-R0SA cell is also shown (arrowhead). D, Triple labeling of TUNEL (red), anti-GFP (green, cells from FVB-GFP mice) and anti- $\beta$-gal (blue, cells from B6-ROSA mice) in a chimera $48 \mathrm{~h}$ after KA injection shows TUNEL-positive cells in both genotypically FVB-GFP and B6-ROSA neurons. Red TUNEL ${ }^{+}$cells are colabeled with green and are genotypically FVB (arrows) or with blue and are B6-R0SA cells (arrowheads). Scale bars: $A, 500 \mu \mathrm{m}$; $B-D, 50 \mu \mathrm{m}$.

\section{Determination of percentage chimerism}

A reasonable but rough estimate of the percentage of cells of each genotype can be obtained by coat color chimerism. As FVB-GFP mice are albino and B6-ROSA mice are non-agouti (black), coat color chimerism is easily ascertained before animals are killed. Staining hippocampal tissue for X-gal or GFP is a more proximal way to estimate hippocampus chimerism in each mouse. This estimate of percentage of chimerism provided an initial readout of chimerism. A cell-based assessment of percentage chimerism in animals that had received KA $48 \mathrm{~h}$ before they were killed was achieved by counting the number of GFP-positive or $\beta$-gal-positive pyramidal neurons (both TUNEL positive and negative) in a defined region of CA1 or CA3 and dividing by the total number of pyramidal cells in that region. The same calculations were made for the dentate gyrus granule cells that do not demonstrate marked cell death at $48 \mathrm{~h}$ post-KA. The chimerism of CA1 and CA3 pyramidal cells and dentate gyrus granule cells was determined for each side of the hippocampal formation. The results indicated that overall chimerism of CA1 and CA3 were similar to the chimerism in the dentate gyrus granule cells on the same side. The correlation coefficients were $0.64-0.86$ for the comparison between the dentate gyrus and CA1 and CA3, respectively. Although this was an overall comparison and not based on specific subregions of the hippocampal fields and the dentate gyrus, we felt comfortable using the dentate gyrus as a cross-check of the chimerism found in CA1 and CA3 after kainic acid to demonstrate that there was no major loss of the GFP marker in pyramidal neurons and provide assurance that our estimates of chimerism were relatively accurate for the pyramidal cell populations $48 \mathrm{~h}$ after KA.

\section{Semiquantification of percentage of dying neurons}

All slides were coded and randomly arranged for counting and analysis in a blinded fashion. Semiquantitative assessments of the right and left hippocampi were initially considered independent as the percentages of chimerism between the right and left sides may vary (Martin et al., 2002). Counts were obtained for the number of surviving and dead/dying $\left(\mathrm{TUNEL}^{+}\right.$) pyramidal cells. Triple-stained (TUNEL, anti-GFP, and TOTO3) tissue was used to determine the numbers of pyramidal cells 

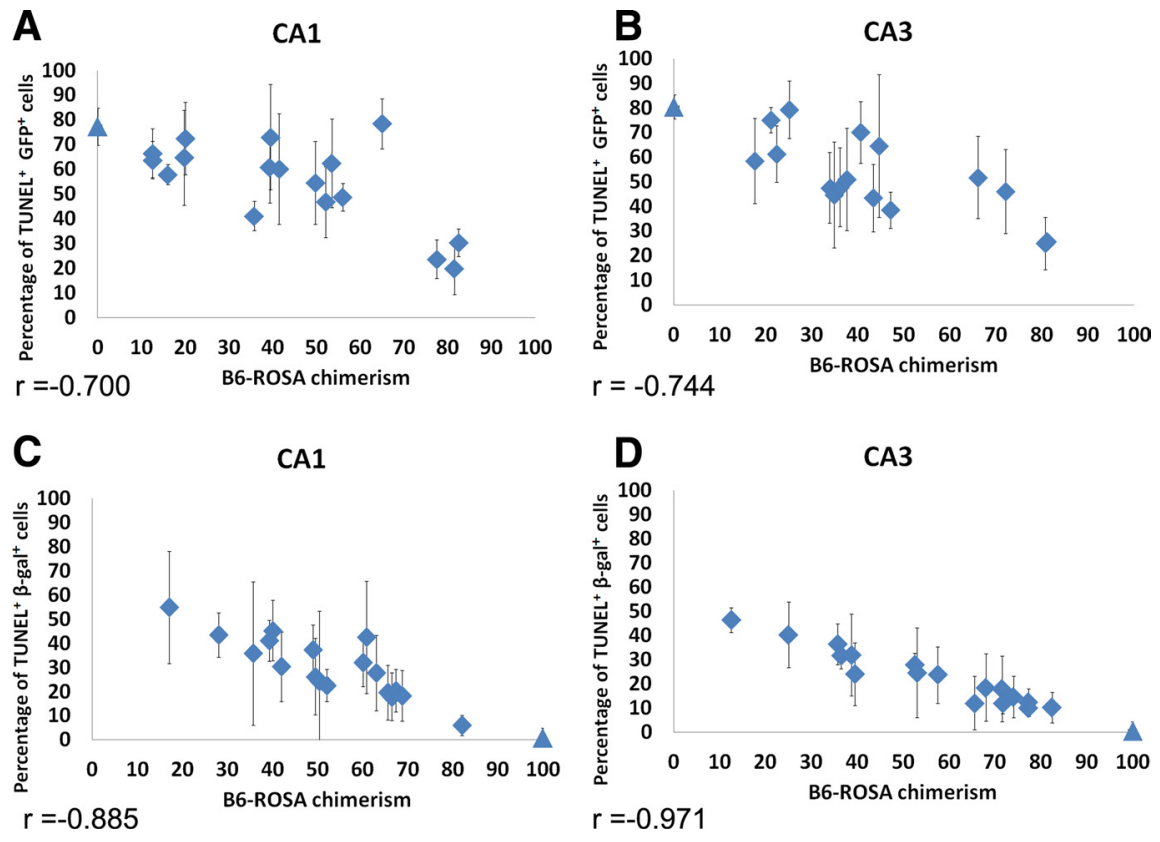

Figure 4. Analysis of chimeric hippocampi demonstrate that the death of resistant B6-ROSA cells and the survival of susceptible FVB-GFP pyramidal cells correlates with the presence of cells of the opposing genotype. $\boldsymbol{A}, \boldsymbol{B}$, The percentage of TUNEL ${ }^{+}$, dying FVB-GFP cells in chimeras in animals with different levels of B6-ROSA chimerism. The percentage of dying cells shows a negative correlation with the B6-ROSA chimerism in CA1 and CA3 regions ( -0.700 and -0.774 , respectively) indicating that, when the B6-ROSA chimerism is increased, fewer GFP/TUNEL ${ }^{+}$neurons are detected. C, D, The number of dying cells from the B6-ROSA genotype increases as the percentage of B6-ROSA-chimerism decreases (i.e., more GFP/FVN cells in the chimera). The percentage of dying B6-ROSA cells is negatively correlated with the percentage B6-ROSA chimerism in CA1 and CA3 $(-0.885$ and -0.971 , respectively). This indicates that with an increase in the percentage of FVB-GFP cells in a chimera, there is an increase in the percentage of B6-ROSA pyramidal cells that will show degeneration. For $\boldsymbol{A}-\boldsymbol{D}$, individual chimeras are denoted by diamonds while the mean of control, non-chimeric mice are shown in the triangles.

that were undergoing cell death from the FVB-GFP genotype. Tissue triple-stained for TUNEL, anti- $\beta$-gal, and TOTO3 was used to determine cell dying/death from the B6-ROSA genotype. There was attrition of sections due to the rigors of triple staining, but at least two sections from different levels of the hippocampus were used for analysis from each mouse. Counts were made of pyramidal neurons in the well defined CA1 and $\mathrm{CA} 3$ regions of the hippocampal formation. A sampling window of $134 \times 134 \mu \mathrm{m}^{2}$ was used and counts made with the aid of a $40 \times$ objective with $2 \times$ zoom. Typically, two windows from each CA1 and CA3 region were selected for counting from each section.

Data were collected in two ways. First, the right and left hippocampi of each mouse were the units of analysis with the mean calculation of dying cells and chimerism made across sections. Second, because the colonization of one region to another in a single hippocampus in the chimeric brain can be different (Martin et al., 2002), we treated each sampling window independently. The Pearson moment correlation statistic was used to assess the relationship between the level of cell death in cells of a particular genotype and the percentage chimerism. Additionally, a cluster analysis was performed using SPSS to identify any subgroups of the data from the window analysis. To further investigate whether different local microenvironments within a chimera have differential effects, we pooled data into three groups: low (B6ROSA $<30 \%$ ), medium (B6-ROSA 31-69\%), and high (B6-ROSA $>70 \%$ ) based on the percentage of B6-ROSA cells within each window. These data were analyzed with a one-way ANOVA, SigmaStat (Jandel Scientific), and intergroup differences were analyzed by Newman-Keuls post hoc test. $p<$ 0.05 was used as the cutoff for a significant difference.

\section{Results}

The FVB-GFP and B6-ROSA transgenic strains have similar sensitivity to KA-induced neuronal degeneration as the nontransgenic parental strains from which they arose

The transgenic reporter lines that contained the $\beta$-gal or the GFP genes provided excellent markers of hippocampal pyramidal cells in both CA1 and CA3, as well as other cells of the hippocampal formation (Fig. 2A,B). It was critical to determine that the genetic endowment of sensitivity or resistance to KA was maintained in the cells of these transgenic lines of mice. B6-ROSA and FVB-GFP non-chimeric control mice received KA and were processed for the presence of TUNEL ${ }^{+}$cells $48 \mathrm{~h}$ after injection. Few, if any, TUNEL ${ }^{+}$cells were seen in the B6-ROSA hippocampus while numerous TUNEL ${ }^{+}$cells were found in the FVB-GFP hippocampus (Fig. 2C,D). Using TUNEL staining we determined that between 70 and $80 \%$ of the hippocampal pyramidal cells in CA1 and CA3 were TUNEL $^{+}$in the FVB-GFP hippocampus (Fig. 2E). In sections stained with TUNEL and GFP, we found that $~ 95 \%$ TUNEL $^{+}$ neurons also coexpressed GFP in CA1 and CA3 (Fig. 2F). In the B6-ROSA hippocampus, there were very few dying cells. Thus, the phenotypes of KA susceptibility in FVB mice and KA resistance in B6 mice were retained in the transgenic reporter lines. In addition, almost all cells that were $\mathrm{TUNEL}^{+}$still express the GFP- or $\beta$-gal (data not shown) genotype marker.

\section{Analysis of chimeras}

\section{Demonstration of cell genotype}

and phenotype

We generated a total of 27 FVB-GFP $\leftrightarrow$ B6-ROSA chimeras (Fig. 3A). Of these 27, three chimeras were used as controls and injected with saline. Of the 24 chimeras that received KA injection, six chimeras died during the KA-induced seizure. This death rate of $25 \%$ is similar to the death rate of FVB or B6 mice in our control work. Two chimeras had seizures that lasted $<1 \mathrm{~h}$ and did not reach criteria (see Materials and Methods) and were excluded from further analysis. Finally, three chimeras were 100\% FVB-GFP or B6-ROSA and were treated as controls. Thus, a total of 13 chimeras were analyzed in this study to determine the role of cell genotype on the cellular phenotype of death. In these chimeras the genotype of the FVB-GFP and the B6-ROSA components was clearly demonstrable using a single label or in double-labeling with antibodies to eGFP and $\beta$-gal (Fig. $3 B$ ). Furthermore, this histological processing allowed us to examine cell genotype ( $\beta$-gal or GFP) and cell phenotype (i.e., TUNEL $^{+}$cells). Confocal serial sections enable us to identify single cells labeled with $\beta$-gal and colabeled with TUNEL (Fig. $3 C 1-C 3 ; \beta$-gal is shown in this figure). TUNEL-positive dying/ dead cells in chimeras were also examined using the rhodamine label (Fig. 3D) and yielded the same results as seen with the fluorescein label.

\section{Analysis of each chimera's hippocampus}

When we examined both the phenotype of cell death and the genotype of cells in the hippocampus of kainate-treated chimeras (Fig. 4), the data pointed to two intriguing conclusions. First, there is less overall death of genetically FVB-GFP pyramidal neurons in either CA1 or CA3 in the presence of B6-ROSA neurons (Fig. $4 A, B$, respectively). Furthermore, with an increasing percentage of the resistant, B6-ROSA neurons in the hippocampus 
there was a concomitant graded reduction in the percentage of FVB-GFP pyramidal neurons that exhibited cell death (Fig. $4 A, B)$. The control data had indicated that $\sim 80 \%$ of all FVB-GFP neurons should be TUNEL $^{+} 48 \mathrm{~h}$ after kainic acid treatment. However, in the chimeric situation, there were as few as $30 \%$ of FVBGFP neurons that were TUNEL $^{+}$in a chimera when a majority of pyramidal neurons were of the resistant, B6ROSAgenotype (e.g., 80\%). Second, the death of normally resistant B6-ROSA neurons is seen in these chimeric hippocampi (Fig. 4C,D). Furthermore, this death of genetically B6-ROSA neurons increased in prevalence when there was an increasing percentage of FVB-GFP neurons in the pyramidal cell layer (Fig. $4 C, D)$. Thus, while the death of genetically B6-ROSA CA1 or CA3 neurons is rare in the control, in the KA-treated B6ROSA hippocampus there is an increase in B6-ROSA cell death that goes from $\sim 20 \%$ to $70 \%$ in a linear relationship as the presence of FVB-GFP neurons increased. The results of both the saving of FVB-GFP neurons and the increased death of B6-ROSA neurons were quite striking and provided strong support for a cell-extrinsic component of KA-induced hippocampal pyramidal cell death. Of note, however, even in the highest percentage of B6-ROSA chimeras $(\sim 80 \%)$ we did not see total rescue of FVB-GFP cells in CA1 or CA3; there was at least $20 \%$ of the FVB-GFP pyramidal cells that were still undergoing cell death (Fig. 4A,B). The persistence of the phenotype was even more striking in the B6-ROSA population where even when there was over 90\% FVB-GFP cells, we never found B6-ROSA cell death to be $>70 \%$ of the total B6ROSA pyramidal neurons in CA1 or CA3 (Fig. 4C,D).

\section{Analysis of the microenvironment of pyramidal cells across chimeric hippocampi}

A point that came to our attention in this analysis was that, in some animals, we found large SDs in the individual animal data (illustrated in Fig. $4 A-D$; see Materials and Methods) that was due to the variability in one sampling window compared with another window in the same animal. Previously, we had found that the percentage of chimerism can differ from one region of the hippocampus to another (Martin et al., 2002). Thus, we reanalyzed the data by examining individual sampling windows across chimeras and treating each as a separate data point that
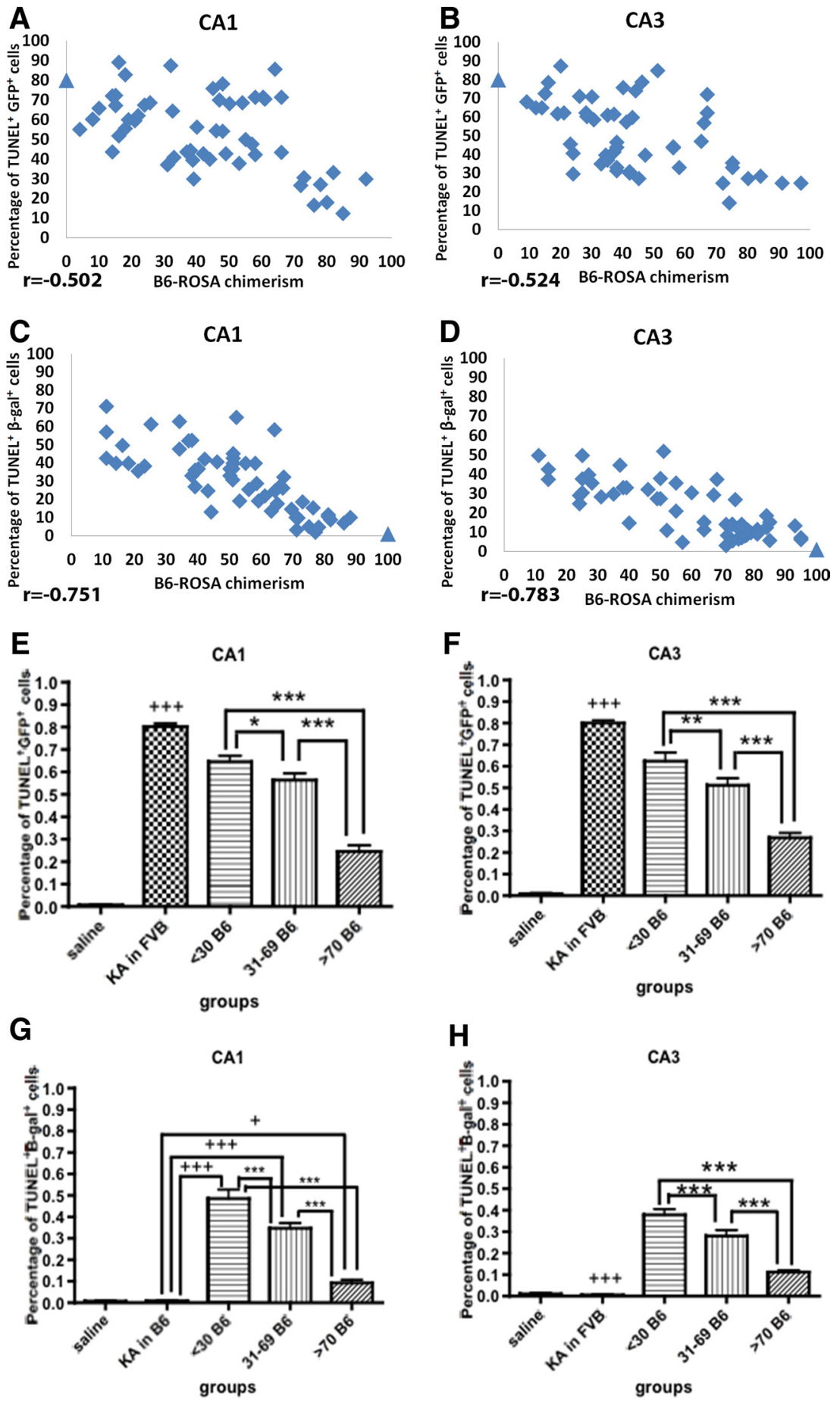

Figure 5. Analysis of individual windows across chimeras demonstrates that susceptibility to KA can be ascribed to microdomains with differing percentages of chimerism. The percentage of TUNEL ${ }^{+}$cells costained with anti-GFP (FVB-GFP dying cells) or anti- $\beta$-gal (B6-ROSA dying cells) are calculated for each window $(134 \times 134 \mu \mathrm{m})$ in the CA1 and CA3 regions from all 13 chimeras, with $\sim 4$ windows sampled from the right and left sides of each chimera. $A-D$, The percentage of cell death is plotted against the percentage chimerism in the B6-ROSA cells. $A, B$, The percentage of TUNEL ${ }^{+}$cells of the FVB-GFP genotype is shown for CA1 and CA3, respectively. The percentage of FVB-GFP cell loss is significantly $(p<0.0001)$ inversely correlated with the percentage of B6-ROSA cells such that there is lower cell death in windows with higher percentage of B6-ROSA cells. $C-D$, The percentage of B6-ROSA TUNEL ${ }^{+}$cells is significantly $(p<0.0001)$ negatively correlated with the percentage of B6-ROSA chimerism seen across windows. B6-ROSA cells show increased degeneration in high percentage of FVB-GFP cells (i.e., in lower percentage of B6-ROSA cells), especially in high percentage FVB-GFP chimeras. $\boldsymbol{E}-\boldsymbol{H}$, Data are binned into three groups based on the percentage of B6-ROSA chimerism $(<30 \%, 31-69 \%,>70 \%)$. $E$ and $\boldsymbol{F}$ show the level of cells death of FVB-GFP cells across the 3 groups. FVB-GFP cells show less TUNEL-positive cell death in windows with higher B6-ROSA chimerism. $\boldsymbol{G}$ and $\boldsymbol{H}$ show the level of cell death in B6-ROSA cells across the 3 groups. More B6-ROSA cells show TUNEL ${ }^{+}$staining in low B6-ROSA (high FVB-GFP) windows; however, TUNEL $^{+}$and $\beta$-gal ${ }^{+}$cells never reach $>70 \%$ of B6-ROSA population. ${ }^{+, *} p<0.05,{ }^{++, * *} p<0.01,{ }^{+++, * * *} p<0.001$. 

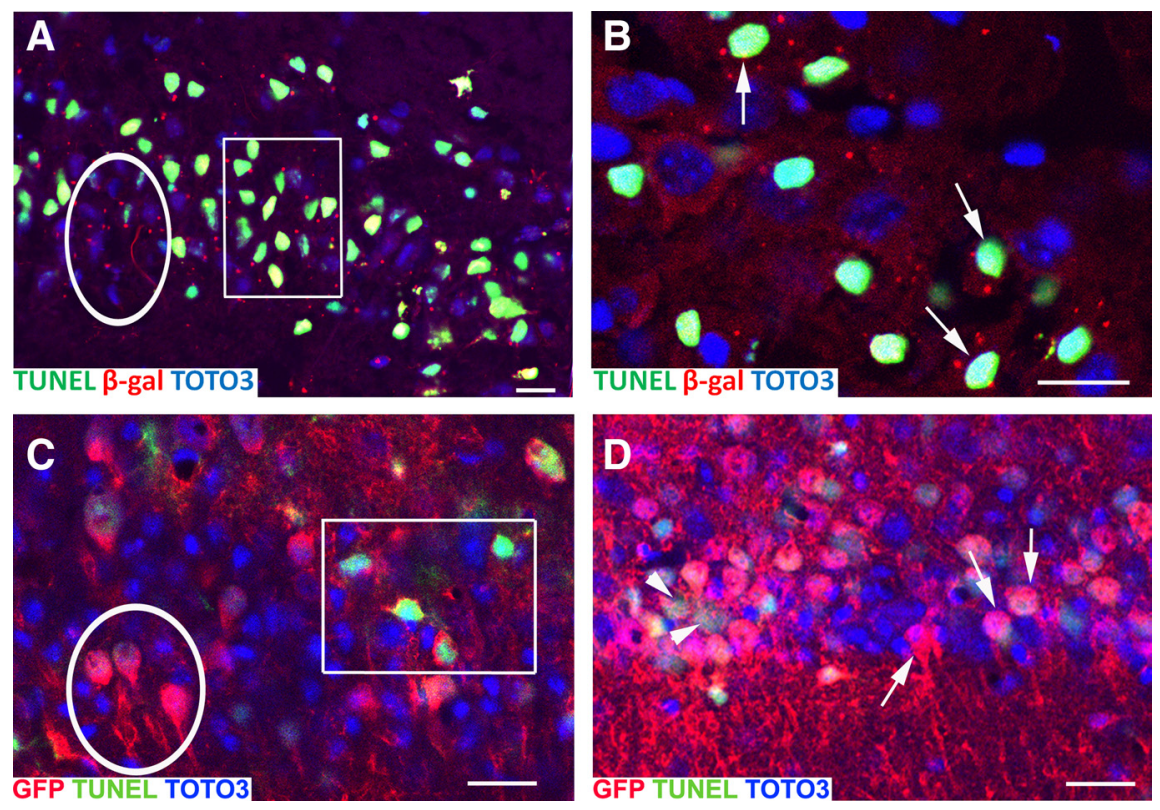

Figure 6. Within each chimera, the susceptibility of B6-ROSA and FVB-GFP hippocampal pyramidal neurons to kainic acid treatment is affected by their local milieu. In all panels, neurons are labeled with T0T03 (blue) while cells undergoing cell death are labeled with TUNEL (green). $\boldsymbol{A}$ and $\boldsymbol{B}$ show B6-ROSA labeled with $\beta$-gal (red puncta), while $\boldsymbol{C}$ and $\boldsymbol{D}$ show FVB-GFP cells labeled with anti-GFP immunocytochemistry (red). $A, A 65 \%$ B6-ROSA chimera is illustrated. Cells in the oval area are a group of exclusively B6-ROSA cells that shows no signs of TUNEL ${ }^{+}$staining. In contrast, cells in the rectangle contain a mixed region of FVB-GFP and B6-ROSA cells with TUNEL-positivity observed in neurons of both genotypes. $B, A$ chimera with equal percentages of B6-ROSA26 and FVB-GFP neurons is illustrated. This higher-magnification image shows some TUNEL ${ }^{+}$nuclei in neurons of both genotypes. Green nuclei surrounded by red puncta indicate dying cells from the B6-R0SA strain (arrows). C, A chimera with 90\% B6-R0SA cells is illustrated. The oval in ( shows GFP-positive FVB-GFP cells that are not TUNEL-positive, apparently protected when surrounded by B6-ROSA cells. The rectangle shows FVB-GFP dying cells when there are fewer B6-ROSA cells present. $\boldsymbol{D}$, In a largely B6-ROSA chimera (estimated at 85\% B6-ROSA), a region enriched in FVB-GFP neurons is shown. When there are surrounding B6-ROSA cells, the FVB-GFP neurons appear to be protected (anti-GFP in red, against the pan-cellular blue staining of TOT03). These cells are scattered among B6-ROSA cells and are not positive for TUNEL (arrows). In contrast, clusters of FVB-GFP cells that are not in the vicinity of B6-ROSA cells do show TUNEL positivity [arrowheads; red + green (TUNEL ${ }^{+}$) + blue]. Scale bar, $50 \mu \mathrm{m}$.

contained the percentage of chimerism as well as the numbers of $\mathrm{TUNEL}^{+}$neurons. Figure 5 illustrates the results of this analysis for the CA1 and CA3 regions when either FVB-GFP or B6-ROSA pyramidal cells were analyzed. This analysis confirmed what we had seen across chimeras. We find decreased levels of cell death in FVB-GFP cells in windows with a high percentage B6-ROSA neurons (Fig. $5 E, F$ ) indicating that the FVB-GFP cells are protected when surrounded by resistant B6-ROSA cells. In contrast, in microenvironments with a high percentage FVB-GFP neurons, increased levels of TUNEL-positive B6-ROSA neurons are observed (Fig. 5G,H), suggesting that the FVB-GFP cells impacted susceptibility of the B6-ROSA neurons to KA. Figure 6 illustrates these points using the $\beta$-gal (Fig. $6 A, B$ ) or the GFP (Fig. 6C,D) marker.

While present in the first analysis, it became more obvious in this analysis that there was a stronger negative correlation between the percentages of B6-ROSA neurons and their cell survival compared with the rescue of FVB-GFP cells (Fig. $5 A, B$ versus $C, D)$, suggesting a stronger bias of the B6-ROSA neuronal genotype in determining the phenotype of cell death. This is also reflected in a tighter range in B6-ROSA cells (i.e., few windows had $>60 \%$ B6-ROSA dying cells in CA1 or $50 \%$ dying cells in CA3 compared with the fuller range of percentages of cell death in the FVB-GFP populations of neurons, Fig. 5C,D). To determine whether there were differences in skewed regions compared with windows that had more even distributions of cell genotypes, a cluster analysis was performed. This analysis detected signifi- cantly different groups and further analysis was conducted using one-way ANOVA which demonstrated that chimeras having $>70 \%$ B6-ROSA cells differed significantly in terms of cell death from those $<70 \%(p<0.001)$ for CA1 and CA3.

\section{Examination of \\ extra-hippocampal structures}

Cell death following kainic acid treatment has previously been observed in a number of structures outside the hippocampus (Benkovic et al., 2006; McLin and Steward, 2006). In our chimeric brains, cell death was also observed in these structures. Qualitative examination suggested that the highest levels of cell death were observed in thalamus, cingulate cortex, entorhinal cortex and hilus of the dentate gyrus, while fewer labeled cells were observed in the amygdala, striatum and few to none were found in the granule cells of the dentate gyrus. In chimeras with increasing levels of FVB-GFP cells there appeared to be an increased TUNEL labeling as the proportion of FVB-GFP cells increased, consistent with the pattern observed from the quantitative analysis of the hippocampus.

\section{Discussion}

Chimeric mice, generated from KA-resistant B6-ROSA and KA-sensitive FVBGFP embryos, were examined to investigate the role of extrinsic and intrinsic factors in determining the degree of susceptibility of hippocampal pyramidal neurons to an excitotoxic administration of kainic acid. Each chimera consists of a variable percentage of cells of each genotype as well as differing percentages of chimerism in different locations within the same animal. The analysis of chimeras exposes clear cellextrinsic and cell-intrinsic influences that determine a pyramidal cell's response to kainic acid, and provides some guiding principles in the effort to parse intrinsic and extrinsic impacts on cell death. First, we show that the differences in KA-induced neurotoxicity are due in good measure to a pyramidal cell extrinsic mechanism. However, we know the cell extrinsic component cannot be a widespread factor (such as a blood-borne molecule) as the "curative" or "damaging" aspect of such a factor would have a more uniform effect and the influence of the cellular composition within a microdomain would be minimal. Instead, we see a microdomain to microdomain difference in cell survival in the same hippocampus that is defined, to some extent, by the percentages of chimerism in those domains. Second, the maximum sensitivity of B6-ROSA neurons to kainic acid rarely goes above $\sim 60 \%$ cell death despite what can be an overwhelming percentage of FVB-GFP neurons in the environs. This suggests that while the environment can modulate the sensitivity, there is a substantial degree of intrinsic resistance of B6-ROSA pyramidal neurons to KA neurotoxicity. Finally, we observe that FVBGFP neurons remain sensitive to KA at least up to a certain level. Our data suggest that when a critical percentage of B6-ROSA cells is reached, putatively $\sim 70 \%$, significant protection is found, suggesting that extrinsic factors can overcome the intrinsic sensitivity but need to reach a threshold level before this impact becomes manifest. 
Thus, from the current analysis, the picture is that while pyramidal cells may have autonomous features that regulate the KA-induced phenotype of cell death, there are robust extrinsic factors that are critical to this phenotype.

What might be some of the intrinsic and extrinsic factors? As kainate neuroexcitoxicity has been shown to be ameliorated by glutamate receptor antagonists (e.g., CNQX, MK-801; Schauwecker, 2010b), an intrinsic suspect would be a strain-specific difference in the levels of kainate, or other excitatory, receptor expression. Schauwecker's group has explored this hypothesis but has found no compelling evidence in this regard (Schauwecker, 2003a,b, 2010b). However, another receptor family, the galanin receptors, has been found to have an intriguing role in hippocampal development and plasticity (Burazin et al., 2000) and several lines of evidence (although the data are somewhat contradictory) support a role for the galanin receptor 1 (GalR1) in neuroprotection in status epilepticus (Mazarati et al., 2004; Schauwecker, 2010a). First, Schauwecker and colleagues (Schauwecker et al., 2004) found a distal region on chromosome 18, that includes the GalR1 locus, houses a quantitative trait locus that significantly influences susceptibility to kainic acid-induced cell death (called, Sicd1). This region was further refined using FVB.B6-Sicd1 lines of mice and the GalR1 locus remained with the region (Lorenzana et al., 2007). Second, recent evidence has demonstrated that GalR1 mRNA is differentially expressed in the hippocampus of sensitive (FVB) and resistant (B6) strains under baseline conditions (Kong et al., 2008; Schauwecker, 2010a). Third, Schauwecker (2010a) provided more direct evidence for the protective role of GalR1 by injecting an antagonist of GalR1 which resulted in increased cell death in the hippocampus following KA in the resistant B6 strain. However, examination of GalR1 $\mathrm{KO}$ mice has given conflicting results with both decreased protection in the KO (Schauwecker, 2010a) or no difference between $\mathrm{KO}$ and wild-type in KA sensitivity (Mazarati et al., 2004). The differences in these two studies may be due to the number of backcrosses to the B6 mouse which would result in differences in the extent of the B6 background strain in the KO. Clearly it will be of interest to evaluate the role of the GalR1 within chimeric mice.

From another perspective, the best documented cases of extrinsic influences on brain neuronal phenotypes involve glia, in particular microglia and astroglia (Ding et al., 2007; Cho et al., 2008). Both microglia and astrocytes show a complex interrelationship with neurons (Ding et al., 2007; Cho et al., 2008). Both astrocytes and microglia are activated in the hippocampus after kainate lesion (Ferraguti et al., 2001; Benkovic et al., 2006). The prevailing evidence for the role of microglia following kainic acid treatment of the hippocampus is that these glia cells aid the neurodegenerative process (Cho et al., 2008; Hong et al., 2010). Whether this effect is mediated through tissue plasminogen activator or tumor necrosis factor $\alpha$ or another molecule, the end result is the involvement of microglia in enhanced hippocampal neuronal death (Zheng et al., 2010; Zhu et al., 2010). On the other hand, astrocytes have been found to have a protective effect, presumably by dampening the excitotoxicity of glutamate from the local environment (Liang et al., 2008). In vivo evidence has indicated a critical role of glia in the kainate-induced neuronal death (Siao et al., 2003; Ding et al., 2007). To date, however, no studies have carefully examined whether the glial reaction to KA insult differs between the sensitive and insensitive strains of mice. Currently we are using the chimeric system to more closely address this issue.

The study of SOD1 mouse models of ALS has provided insights to the non-cell autonomy of neurodegeneration. The neu- ronal degeneration in ALS, traditionally thought to be a primary disorder of motor neurons, was found to be abrogated in mouse models of the disorder when the mutant SOD1 protein was solely expressed in neurons (Pramatarova et al., 2001; Lino et al., 2002). However, a deleterious effect on motorneurons of mutant SOD1 in culture has also been documented (Xiao et al., 2007). The use of experimental mouse chimeras pointed to the other-thanneuronal site of mutant gene action in an analogous manner to the target of kainic acid in our studies (Clement et al., 2003). The targeting of mutant SOD1 allele to astroglia did not produce neuronal pathology (Gong et al., 2000), suggesting that one needed to look beyond neurons and astroglia. However, more recent work from the Cleveland lab demonstrated that if the pathogenic SOD1 mutant protein was diminished in astrocytes that disease progression and microglial activation were slowed (Yamanaka et al., 2008). The role of microglia was supported by work from this group using Cre-targeted expression where mutant SOD1 expression in microglia is an important factor for the progression of neuronal pathology that is seen in the mouse model of ALS (Boillee et al., 2006). In line with the impact of microglia on ALS, a beneficial effect of bone marrow-derived microglia expressing myeloid differentiation factor 88 has been reported (Kang and Rivest, 2007). However, other work has found that the elimination of microglia has little impact on ALS progression in the mouse model (Gowing et al., 2008). Nonetheless, a persuasive case has been made that there is a key role for microglia and astroglia in neurodegenerative disorders where the factor under study is expressed in many cell types that include glia (Lobsiger and Cleveland, 2007).

Whether glial cells are assigned a key factor in the KA-induced death of hippocampal pyramidal cells will depend on similar experimental strategies as performed in the SOD1 mutant model. Nonetheless, the dissection of cell intrinsic and extrinsic mechanisms for kainic acid excitotoxicity is a key first step in the elucidation of the various factors that result in cell death. The multifactorial nature of cell death seems to be emerging as the rule rather than the exception (Ilieva et al., 2009) and the chimeric analysis is a critical means to determine the features of the neuropil that confer resistance to sensitive neurons and provide optimism for interventions in these debilitating conditions.

\section{References}

Benkovic SA, O'Callaghan JP, Miller DB (2006) Regional neuropathology following kainic acid intoxication in adult and aged C57BL/6J mice. Brain Res 1070:215-231.

Boillée S, Yamanaka K, Lobsiger CS, Copeland NG, Jenkins NA, Kassiotis G Kollias G, Cleveland DW (2006) Onset and progression in inherited ALS determined by motor neurons and microglia. Science 312:1389-1392.

Burazin TC, Larm JA, Ryan MC, Gundlach AL (2000) Galanin-R1 and -R2 receptor mRNA expression during the development of rat brain suggests differential subtype involvement in synaptic transmission and plasticity. Eur J Neurosci 12:2901-2917.

Cho IH, Hong J, Suh EC, Kim JH, Lee H, Lee JE, Lee S, Kim CH, Kim DW, Jo EK, Lee KE, Karin M, Lee SJ (2008) Role of microglial IKKbeta in kainic acid-induced hippocampal neuronal cell death. Brain 131:3019-3033.

Clement AM, Nguyen MD, Roberts EA, Garcia ML, Boillée S, Rule M, McMahon AP, Doucette W, Siwek D, Ferrante RJ, Brown RH Jr, Julien JP, Goldstein LS, Cleveland DW (2003) Wild-type nonneuronal cells extend survival of SOD1 mutant motor neurons in ALS mice. Science 302:113-117.

Ding S, Fellin T, Zhu Y, Lee SY, Auberson YP, Meaney DF, Coulter DA, Carmignoto G, Haydon PG (2007) Enhanced astrocytic $\mathrm{Ca}^{2+}$ signals contribute to neuronal excitotoxicity after status epilepticus. J Neurosci 27:10674-10684

Ferraguti F, Corti C, Valerio E, Mion S, Xuereb J (2001) Activated astrocytes in areas of kainate-induced neuronal injury upregulate the expression of the metabotropic glutamate receptors 2/3 and 5. Exp Brain Res 137:1-11. 
Ferraro TN, Golden GT, Smith GG, Berrettini WH (1995) Differential susceptibility to seizures induced by systemic kainic acid treatment in mature DBA/2J and C57BL/6J mice. Epilepsia 36:301-307.

Goldowitz D, Moran TH, Wetts R (1992) Mouse chimeras in the study of genetic and structural determinants of behavior. In: Techniques for the genetic analysis of brain and behavior: focus on the mouse, vol 8 (Goldowitz D, Wahlsten D, Wimer R, eds), pp 271-290. Amsterdam: Elsevier.

Gong YH, Parsadanian AS, Andreeva A, Snider WD, Elliott JL (2000) Restricted expression of G86R Cu/Zn superoxide dismutase in astrocytes results in astrocytosis but does not cause motoneuron degeneration. J Neurosci 20:660-665.

Gowing G, Philips T, Van Wijmeersch B, Audet JN, Dewil M, Van Den Bosch L, Billiau AD, Robberecht W, Julien JP (2008) Ablation of proliferating microglia does not affect motor neuron degeneration in amyotrophic lateral sclerosis caused by mutant superoxide dismutase. J Neurosci 28:10234-10244.

Hadjantonakis AK, Gertsenstein M, Ikawa M, Okabe M, Nagy A (1998) Generating green fluorescent mice by germline transmission of green fluorescent ES cells. Mech Dev 76:79-90.

Hong J, Cho IH, Kwak KI, Suh EC, Seo J, Min HJ, Choi SY, Kim CH, Park SH, Jo EK, Lee S, Lee KE, Lee SJ (2010) Microglial Toll-like receptor 2 contributes to kainic acid-induced glial activation and hippocampal neuronal cell death. J Biol Chem 285:39447-39457.

Hu RQ, Koh S, Torgerson T, Cole AJ (1998) Neuronal stress and injury in C57/BL mice after systemic kainic acid administration. Brain Res 810:229-240.

Ilieva H, Polymenidou M, Cleveland DW (2009) Non-cell autonomous toxicity in neurodegenerative disorders: ALS and beyond. J Cell Biol 187:761-772.

Jensen P, Smeyne R, Goldowitz D (2004) Analysis of cerebellar development in math1 null embryos and chimeras. J Neurosci 24:2202-2211.

Kang J, Rivest S (2007) MyD88-deficient bone marrow cells accelerate onset and reduce survival in a mouse model of amyotrophic lateral sclerosis. J Cell Biol 179:1219-1230.

Kong S, Lorenzana A, Deng Q, McNeill TH, Schauwecker PE (2008) Variation in Galr1 expression determines susceptibility to exocitotoxininduced cell death in mice. Genes Brain Behav 7:587-598.

Liang J, Takeuchi H, Doi Y, Kawanokuchi J, Sonobe Y, Jin S, Yawata I, Li H, Yasuoka S, Mizuno T, Suzumura A (2008) Excitatory amino acid transporter expression by astrocytes is neuroprotective against microglial excitotoxicity. Brain Res 1210:11-19.

Lino MM, Schneider C, Caroni P (2002) Accumulation of SOD1 mutants in postnatal motoneurons does not cause motoneuron pathology or motoneuron disease. J Neurosci 22:4825-4832.

Liu L, Hsu SS, Kalia SK, Lozano AM (2003) Injury and strain-dependent dopaminergic neuronal degeneration in the substantia nigra of mice after axotomy or MPTP. Brain Res 994:243-252.

Lobsiger CS, Cleveland DW (2007) Glial cells as intrinsic components of non-cell-autonomous neurodegenerative disease. Nat Neurosci 10:1355-1360.

Lopez-Picon FR, Kukko-Lukjanov TK, Holopainen IE (2006) The calpain inhibitor MDL-28170 and the AMPA/KA receptor antagonist CNQX inhibit neurofilament degradation and enhance neuronal survival in kainic acid-treated hippocampal slice cultures. Eur J Neurosci 23:2686-2694.

Lorenzana A, Chancer Z, Schauwecker PE (2007) A quantitative trait locus on chromosome 18 is a critical determinant of excitotoxic cell death susceptibility. Eur J Neurosci 25:1998-2008.

Martin LA, Tan SS, Goldowitz D (2002) Clonal architecture of the mouse hippocampus. J Neurosci 22:3520-3530.

Mazarati A, Lu X, Shinmei S, Badie-Mahdavi H, Bartfai T (2004) Patterns of seizures, hippocampal injury and neurogenesis in three models of status epilepticus in galanin receptor type 1 (GalR1) knockout mice. Neuroscience 128:431-441.

McKhann GM 2nd, Wenzel HJ, Robbins CA, Sosunov AA, Schwartzkroin PA
(2003) Mouse strain differences in kainic acid sensitivity, seizure behavior, mortality, and hippocampal pathology. Neuroscience 122:551-561.

McLin JP, Steward O (2006) Comparison of seizure phenotype and neurodegeneration induced by systemic kainic acid in inbred, outbred, and hybrid mouse strains. Eur J Neurosci 24:2191-2202.

Mullen RJ, Whitten WK (1971) Relationship of genotype and degree of chimerism in coat color to sex ratios and gametogenesis in chimeric mice. J Exp Zool 178:165-176.

Ogita K, Okuda H, Yamamoto Y, Nishiyama N, Yoneda Y (2003) In vivo neuroprotective role of NMDA receptors against kainate-induced excitotoxicity in murine hippocampal pyramidal neurons. J Neurochem 85:1336-1346.

Ogita K, Okuda H, Watanabe M, Nagashima R, Sugiyama C, Yoneda Y (2005) In vivo treatment with the $\mathrm{K}+$ channel blocker 4-aminopyridine protects against kainate-induced neuronal cell death through activation of NMDA receptors in murine hippocampus. Neuropharmacology $48: 810-821$.

Pramatarova A, Laganière J, Roussel J, Brisebois K, Rouleau GA (2001) Neuron-specific expression of mutant superoxide dismutase 1 in transgenic mice does not lead to motor impairment. J Neurosci 21:3369-3374.

Racine RJ (1972) Modification of seizure activity by electrical stimulation. II. Motor seizure. Electroencephalogr Clin Neurophysiol 32:281-294.

Schauwecker PE (2002) Modulation of cell death by mouse genotype: differential vulnerability to excitatory amino acid-induced lesions. Exp Neurol 178:219-235.

Schauwecker PE (2003a) Differences in ionotropic glutamate receptor subunit expression are not responsible for strain-dependent susceptibility to excitotoxin-induced injury. Brain Res Mol Brain Res 112:70-81.

Schauwecker PE (2003b) Genetic basis of kainate-induced excitotoxicity in mice: phenotypic modulation of seizure-induced cell death. Epilepsy Res 55:201-210.

Schauwecker PE (2010a) Galanin receptor 1 deletion exacerbates hippocampal neuronal loss after systemic kainate administration in mice. PLoS One 5:e15657.

Schauwecker PE (2010b) Neuroprotection by glutamate receptor antagonists against seizure-induced excitotoxic cell death in the aging brain. Exp Neurol 224:207-218.

Schauwecker PE, Steward O (1997) Genetic determinants of susceptibility to excitotoxic cell death: implications for gene targeting approaches. Proc Natl Acad Sci U S A 94:4103-4108.

Schauwecker PE, Williams RW, Santos JB (2004) Genetic control of sensitivity to hippocampal cell death induced by kainic acid: a quantitative trait loci analysis. J Comp Neurol 477:96-107.

Schori H, Yoles E, Wheeler LA, Raveh T, Kimchi A, Schwartz M (2002) Immune-related mechanisms participating in resistance and susceptibility to glutamate toxicity. Eur J Neurosci 16:557-564.

Siao CJ, Fernandez SR, Tsirka SE (2003) Cell type-specific roles for tissue plasminogen activator released by neurons or microglia after excitotoxic injury. J Neurosci 23:3234-3242.

Soriano P (1999) Generalized lacZ expression with the ROSA26 Cre reporter strain. Nat Genet 21:70-71.

Xiao Q, Zhao W, Beers DR, Yen AA, Xie W, Henkel JS, Appel SH (2007) Mutant SOD1(G93A) microglia are more neurotoxic relative to wild-type microglia. J Neurochem 102:2008-2019.

Yamanaka K, Chun SJ, Boillee S, Fujimori-Tonou N, Yamashita H, Gutmann DH, Takahashi R, Misawa H, Cleveland DW (2008) Astrocytes as determinants of disease progression in inherited amyotrophic lateral sclerosis. Nat Neurosci 11:251-253.

Zheng H, Zhu W, Zhao H, Wang X, Wang W, Li Z (2010) Kainic acidactivated microglia mediate increased excitability of rat hippocampal neurons in vitro and in vivo: crucial role of interleukin-1beta. Neuroimmunomodulation 17:31-38.

Zhu W, Zheng H, Shao X, Wang W, Yao Q, Li Z (2010) Excitotoxicity of TNFalpha derived from KA activated microglia on hippocampal neurons in vitro and in vivo. J Neurochem 114:386-396. 